Research Article

\title{
Definite Integrals Involving Combinations of Powers and Logarithmic Functions of Complicated Arguments Expressed in Terms of the Hurwitz Zeta Function
}

\author{
Robert Reynolds $(\mathbb{D}$ and Allan Stauffer \\ Department of Mathematics and Statistics, York University, Toronto, Ontario M3J1P3, Canada \\ Correspondence should be addressed to Robert Reynolds; milver@my.yorku.ca
}

Received 11 March 2021; Accepted 4 May 2021; Published 11 May 2021

Academic Editor: Elena Guardo

Copyright ( 2021 Robert Reynolds and Allan Stauffer. This is an open access article distributed under the Creative Commons Attribution License, which permits unrestricted use, distribution, and reproduction in any medium, provided the original work is properly cited.

\begin{abstract}
In this manuscript, the authors derive closed formula for definite integrals of combinations of powers and logarithmic functions of complicated arguments and express these integrals in terms of the Hurwitz zeta functions. These derivations are then expressed in terms of fundamental constants, elementary, and special functions. A summary of the results is produced in the form of a table of definite integrals for easy referencing by readers.
\end{abstract}

\section{Introduction}

In this manuscript, the authors derive the definite integrals given by

$$
\int_{0}^{1} \frac{\log \left(x^{m}+1\right)\left(\log ^{k}(a / x)+\log ^{k}(a x)\right)}{x} \mathrm{~d} x
$$

and

$$
\int_{0}^{1} \frac{\log \left(1-x^{n}\right)\left(\log ^{k}(a / x)+\log ^{k}(a x)\right)}{x} \mathrm{~d} x,
$$

in terms of the Hurwitz zeta function, where the parameters $k, a, m$, and $n$ are general complex numbers. A summary of the results is given in a table of integrals for easy reading. This work is important because the authors were unable to find similar results in current literature. Tables of definite integrals provide a useful summary and reference for readers seeking such integrals for potential use in their research. This work looks at definite integrals of the hyperbolic arctangent function and the product of logarithmic functions with complicated arguments and powers. We use our simultaneous contour integration method to aid in our derivations of the closed forms solutions in terms of the Hurwitz zeta function, which provides analytic continuation of the results.
The derivations follow the method used by us in [1]. The generalized Cauchy's integral formula is given by

$$
\frac{y^{k}}{k !}=\frac{1}{2 \pi i} \int_{C} \frac{e^{w y}}{w^{k+1}} \mathrm{~d} w,
$$

where $C$ is in general an open contour in the complex plane where the bilinear concomitant has the same value at the end points of the contour. This method involves using a form of equation (3), then multiplying both sides by a function, and then taking a definite integral of both sides. This yields a definite integral in terms of a contour integral. A second contour integral is derived by multiplying equation (3) by different function and summing performing some substitutions so that the contour integrals are the same.

\section{Derivation of the First Contour Integral}

We use the method in [1]. Using a generalization of Cauchy's integral formula equation (3), we will form two equations and add them together. For the first and second equations, replace $y$ by $\log (a x)$ and $y$ by $\log (a / x)$, respectively. Next, we add these equations followed by multiplying both sides by $\left(\log \left(x^{m}+1\right) / x\right)$ and taking the definite integral over $x \in[0,1]$ to get 


$$
\begin{aligned}
& \frac{1}{k !} \int_{0}^{1} \frac{\log \left(x^{m}+1\right)\left(\log ^{k}(a / x)+\log ^{k}(a x)\right)}{x} \mathrm{~d} x \\
& =\frac{1}{2 \pi i} \int_{0}^{1} \int_{C} a^{w} w^{-k-1} x^{-w-1}\left(x^{2 w}+1\right) \log \left(x^{m}+1\right) \mathrm{d} w \mathrm{~d} x \\
& =\frac{1}{2 \pi i} \int_{C} \int_{0}^{1} a^{w} w^{-k-1} x^{-w-1}\left(x^{2 w}+1\right) \log \left(x^{m}+1\right) \mathrm{d} x \mathrm{~d} w \\
& =\frac{1}{2 \pi i} \int_{C} a^{w} w^{-k-3}\left(\pi w \csc \left(\frac{\pi w}{m}\right)-m\right) \mathrm{d} w,
\end{aligned}
$$

from equation (2.6.19.6) in [2] and the integral is valid for $a$, $m$, and $k$ complex and $-1<\operatorname{Re}(w)<0$ where the logarithmic function is defined in equation (4.1.2) in [3].

\section{Derivation of the Second Contour Integral}

Using a generalization of Cauchy's integral formula equation (3), we will form two equations and add them together. For the first and second equations, replace $y$ by $\log (a x)$ and $y$ by $\log (a / x)$, respectively. Next, we add these equations followed by multiplying both sides by $\left(\log \left(1-x^{n}\right) / x\right)$ and taking the definite integral over $x \in[0,1]$ to get

$$
\begin{aligned}
& \frac{1}{k !} \int_{0}^{1} \frac{\log \left(1-x^{n}\right)\left(\log ^{k}(a / x)+\log ^{k}(a x)\right)}{x} \mathrm{~d} x \\
& =\frac{1}{2 \pi i} \int_{0}^{1} \int_{C} a^{w} w^{-k-1} x^{-w-1}\left(x^{2 w}+1\right) \log \left(1-x^{n}\right) \mathrm{d} w \mathrm{~d} x \\
& =\frac{1}{2 \pi i} \int_{C} \int_{0}^{1} a^{w} w^{-k-1} x^{-w-1}\left(x^{2 w}+1\right) \log \left(1-x^{n}\right) \mathrm{d} x \mathrm{~d} w \\
& =\frac{1}{2 \pi i} \int_{C} a^{w} w^{-k-3}\left(\pi w \cot \left(\frac{\pi w}{n}\right)-n\right) \mathrm{d} w,
\end{aligned}
$$

from equation (2.6.19.6) in [2] where $-1<\operatorname{Re}(w)<0$.

\section{Derivation of the Infinite Sum of the First Contour Integral}

Again, using the method in [1] and equation (3), we replace $y$ by $\log (a)+(i \pi(2 y+1) / m)$, multiply both sides by $-2 \pi i$, replace $k$ by $k+1$, and take the infinite sum of both sides over $y \in[0, \infty)$ simplifying in terms of the Hurwitz zeta function to get

$$
\begin{aligned}
& -\frac{i(2 \pi)^{k+2}(i / m)^{k+1} \zeta(-k-1,(\pi-i m \log (a) / 2 \pi))}{(k+1) !} \\
& =-\frac{1}{2 \pi i} \sum_{y=0}^{\infty} \int_{C} 2 i \pi w^{-k-1} e^{w(\log (a)+(i \pi(2 y+1) / m))} \mathrm{d} w \\
& =-\frac{1}{2 \pi i} \int_{C} \sum_{y=0}^{\infty} 2 i \pi w^{-k-1} e^{w(\log (a)+(i \pi(2 y+1) / m))} \mathrm{d} w \\
& =\frac{1}{2 \pi i} \int_{C} \pi a^{w} w^{-k-2} \csc \left(\frac{\pi w}{m}\right) \mathrm{d} w,
\end{aligned}
$$

from equation (1.232.3) in [4] where $\operatorname{csch}(i x)=i \csc (x)$ from (4.5.10) in [3] and $\operatorname{Im}(w)>0$ for the convergence of the sum. We use equation (9.521.1) in [4], where $\zeta(s, u)$ is the Hurwitz zeta function.

\section{Derivation of the Infinite Sum of the Second Contour Integral}

Again, using the method in [1] and equation (3), we replace $y$ by $\log (a)+(2 i \pi(y+1) / n)$, multiply both sides by $-2 \pi i$, replace $k$ by $k+1$, and take the infinite sum of both sides over $y \in[0, \infty)$ simplifying in terms of the Hurwitz zeta function to get

$$
\begin{aligned}
& -\frac{i(2 \pi)^{k+2}(i / n)^{k+1} \zeta(-k-1,1-(i n \log (a) / 2 \pi))}{(k+1) !} \\
& =-\frac{1}{2 \pi i} \sum_{y=0}^{\infty} \int_{C} 2 i \pi w^{-k-1} e^{w(\log (a)+(2 i \pi(y+1) / n))} \mathrm{d} w \\
& =-\frac{1}{2 \pi i} \int_{C} \sum_{y=0}^{\infty} 2 i \pi w^{-k-1} e^{w(\log (a)+(2 i \pi(y+1) / n))} \mathrm{d} w \\
& =\frac{1}{2 \pi i} \int_{C} \pi a^{w} w^{-k-2} \cot \left(\frac{\pi w}{n}\right)+i \pi a^{w} w^{-k-2} \mathrm{~d} w,
\end{aligned}
$$

from equation (1.232.1) in [5].

\section{Derivation of the Additional Contours}

Again, using the method in [1] and equation (3), we replace $y$ by $\log (a), k$ by $k+1$, multiply both sides by $\pi i$, and simplify to get

$$
\frac{i \pi \log ^{k+1}(a)}{(k+1) !}=\frac{1}{2 \pi i} \int_{C} i \pi a^{w} w^{-k-2} \mathrm{~d} w .
$$


Again, using the method in [1] and equation (3), we replace $y$ by $\log (a), k$ by $k+2$ and multiply both sides by $-n$ simplify to get

$$
-\frac{n \log ^{k+2}(a)}{(k+2) !}=-\frac{1}{2 \pi i} \int_{C} n a^{w} w^{-k-3} \mathrm{~d} w .
$$

Again, using the method in [1] and equation (3), we replace $y$ by $\log (a), k$ by $k+2$, multiply both sides by $-m$, and simplify to get

$$
-\frac{m \log ^{k+2}(a)}{(k+2) !}=-\frac{1}{2 \pi i} \int_{C} m a^{w} w^{-k-3} \mathrm{~d} w .
$$

\section{Derivation of the Definite Integrals in Terms of the Hurwitz Zeta Function}

Since the right-hand side of equations (4) and (5) are equal to the sum of the right-hand sides of equations (6)-(10), we can equate the left-hand sides simplifying the factorials to get

$$
\begin{aligned}
& \int_{0}^{1} \frac{\log \left(x^{m}+1\right)\left(\log ^{k}(a / x)+\log ^{k}(a x)\right)}{x} \mathrm{~d} x \\
& =-\frac{m \log ^{k+2}(a)}{(k+1)(k+2)}-\frac{i(2 \pi)^{k+2}(i / m)^{k+1} \zeta(-k-1,(\pi-i m \log (a) / 2 \pi))}{k+1},
\end{aligned}
$$

$$
\begin{aligned}
\int_{0}^{1} & \frac{\log \left(1-x^{n}\right)\left(\log ^{k}(a / x)+\log ^{k}(a x)\right)}{x} \mathrm{~d} x \\
= & -\frac{i(2 \pi)^{k+2}(i / n)^{k+1} \zeta(-k-1,1-(i n \log (a) / 2 \pi))}{k+1} \\
& -\frac{n \log ^{k+2}(a)}{(k+1)(k+2)}-\frac{i \pi \log ^{k+1}(a)}{k+1} .
\end{aligned}
$$

\section{Derivation of Logarithmic and Hyperbolic Tangent Integrals in Terms of the Hurwitz Zeta Function}

Using equations (11) and (12) and taking their difference simplifying, we get

$$
\begin{aligned}
& \int_{0}^{1} \frac{\tanh ^{-1}\left(x^{m}\right)\left(\log ^{k}(a / x)+\log ^{k}(a x)\right)}{x} \mathrm{~d} x \\
& =\frac{(2 \pi)^{k+2}(i / m)^{k}}{2(k+1) m}\left(\zeta\left(-k-1, \frac{\pi-i m \log (a)}{2 \pi}\right)\right. \\
& \left.-\zeta\left(-k-1,1-\frac{i m \log (a)}{2 \pi}\right)\right) \\
& +i \pi m \log ^{k+1}(a) .
\end{aligned}
$$

Using equations (11) and (12) and adding them and then simplifying, we get

$$
\begin{aligned}
& \int_{0}^{1} \frac{\left(\log ^{k}(a / x)+\log ^{k}(a x)\right) \log \left(\left(x^{m}+1\right)\left(1-x^{n}\right)\right)}{x} \mathrm{~d} x \\
& =\frac{4 \pi^{2}}{(k+1)(k+2) m n}\left((2 \pi)^{k} k+2^{k+1} \pi^{k}\right) \\
& \left(n\left(\frac{i}{m}\right)^{k} \zeta\left(-k-1, \frac{\pi-i m \log (a)}{2 \pi}\right)\right. \\
& \left.+m\left(\frac{i}{n}\right)^{k} \zeta\left(-k-1,1-\frac{i n \log (a)}{2 \pi}\right)\right) \\
& \quad-m n \log ^{k+1}(a)(\log (a)(m+n)+i \pi(k+2)) .
\end{aligned}
$$

\section{Derivation of Logarithmic and Hyperbolic Arctangent Integrals in Terms of the Zeta Function}

Using equations (13) and (14) and setting $a=1$ simplifying, we get

$$
\begin{aligned}
& \int_{0}^{1} \frac{\tanh ^{-1}(x) \log ^{k}(x)}{x} \mathrm{~d} x=2^{-k-2}\left(2^{k+2}-1\right) e^{i \pi k} \zeta(k+2) \Gamma(k+1), \\
& \int_{0}^{1} \frac{\log ^{k}(x) \log \left(\left(x^{m}+1\right)\left(1-x^{n}\right)\right)}{x} \mathrm{~d} x=\frac{1}{2} e^{i \pi k} \zeta(k+2) \Gamma(k+1)\left(\left(2-2^{-k}\right) m^{-k-1}-2 n^{-k-1}\right),
\end{aligned}
$$

from entry (2) in the table below (64:7) in [5]. 


\section{Derivation of Logarithmic and Hyperbolic Arctangent Integrals in Terms of the Log Gamma Function}

Using equations (13) and (14), replacing $a$ by $e^{a i}$, applying L'Hopital's rule to the right-hand side as $k \longrightarrow-1$, respectively, and simplifying, we get

$$
\begin{aligned}
& \int_{0}^{1} \frac{\tanh ^{-1}\left(x^{m}\right)}{x\left(a^{2}+\log ^{2}(x)\right)} \mathrm{d} x \\
& =\frac{\pi}{4 a} \log \left(\frac{a m \Gamma(a m / 2 \pi)^{2}}{2 \pi \Gamma(a m+\pi / 2 \pi)^{2}}\right), \\
& \int_{0}^{1} \frac{\log \left(\left(x^{m}+1\right)\left(1-x^{n}\right)\right)}{x\left(a^{2}+\log ^{2}(x)\right)} d x \\
& =\frac{1}{2 a}\left(-2 \pi \log \left(\Gamma\left(\frac{a m+\pi}{2 \pi}\right) \Gamma\left(\frac{a n}{2 \pi}+1\right)\right)-a m\right. \\
& \quad+a m \log (i a)-a m \log \left(\frac{2 i \pi}{m}\right)-a n+a n \log (i a) \\
& \left.-a n \log \left(\frac{2 i \pi}{n}\right)+\pi \log (i \pi a)-\pi \log \left(\frac{i}{2 n}\right)\right),
\end{aligned}
$$

from equation $(64: 10: 2)$ in [5].

\section{Derivation of Logarithmic and Hyperbolic Arctangent Integrals in Terms of the Digamma Function}

Using equations (13) and (14), replacing $a$ by $e^{a i}$, applying L'Hopital's rule to the right-hand side as $k \longrightarrow-2$, respectively, and simplifying, we get

$$
\begin{aligned}
& \int_{0}^{1} \frac{\left(a^{2}-\log ^{2}(x)\right) \tanh ^{-1}\left(x^{m}\right)}{x\left(a^{2}+\log ^{2}(x)\right)^{2}} \mathrm{~d} x \\
& =\frac{1}{4}\left(-m \psi^{(0)}\left(\frac{a m}{2 \pi}+1\right)+m \psi^{(0)}\left(\frac{a m+\pi}{2 \pi}\right)+\frac{\pi}{a}\right), \\
& \int_{0}^{1} \frac{\left(a^{2}-\log ^{2}(x)\right) \log \left(\left(-x^{m}-1\right)\left(x^{n}-1\right)\right)}{x\left(a^{2}+\log ^{2}(x)\right)^{2}} \mathrm{~d} x \\
& =\frac{1}{2 a}\left(-a m \log (i a)+a m \log \left(\frac{i}{m}\right)\right. \\
& +a m \log (2 \pi)+a m \psi^{(0)}\left(\frac{a m+\pi}{2 \pi}\right) \\
& \quad-a n \log (i a)+a n \log \left(\frac{i}{n}\right)+a n \log (2 \pi) \\
& \left.\quad+a n \psi^{(0)}\left(\frac{a n}{2 \pi}+1\right)-\pi\right),
\end{aligned}
$$

from equation $(64: 4: 2)$ in [5].

\section{Derivation of Logarithmic and Hyperbolic Arctangent Integrals in Terms of Fundamental Constants and Special Functions}

In this section, we will derive definite integrals in terms of special functions and fundamental constants such as Euler's constant $(\gamma)$, Catalan's constant $(C)$, Glaisher's constant $(A)$, and $\pi$. This section showcases just a subset of the range of evaluations of these integral formulae.

\subsection{Hyperbolic Tangent Integrals}

Example 1. Using equation (13), replacing $a$ by $e^{-(i \pi / 2 m)}$, setting $k=-3, m=1$, and simplifying, we get

$$
\int_{0}^{1} \frac{\left(\pi^{3}-12 \pi \log ^{2}(x)\right) \tanh ^{-1}(x)}{x\left(4 \log ^{2}(x)+\pi^{2}\right)^{3}} \mathrm{~d} x=\frac{2 C-1}{16 \pi},
$$

from equations (23.2.23) in [3] and $(64: 7: 1)$ in [5].

Example 2. Using equation (16), taking the first partial derivative with respect to $k$, setting $k=0$, and simplifying, we get

$$
\int_{0}^{1} \frac{\log (\log (x)) \tanh ^{-1}(x)}{x} \mathrm{~d} x=\frac{1}{24} \pi^{2}\left(\log \left(\frac{16 \pi^{3}}{A^{36}}\right)+3 i \pi\right)
$$

from equation (A.11) in [6].

Example 3. Using equation (16), setting $k=1 / 2$, and simplifying, we get

$$
\int_{0}^{1} \frac{\sqrt{\log (x)} \tanh ^{-1}(x)}{x} \mathrm{~d} x=-\frac{1}{16} i(\sqrt{2}-8) \sqrt{\pi} \zeta\left(\frac{5}{2}\right) .
$$

Example 4. Using equation (16), setting $k=-1 / 2$, and simplifying, we get

$$
\int_{0}^{1} \frac{\tanh ^{-1}(x)}{x \sqrt{\log (x)}} \mathrm{d} x=\frac{1}{4} i(\sqrt{2}-4) \sqrt{\pi} \zeta\left(\frac{3}{2}\right) .
$$

Example 5. Using equation (16), taking the first partial derivative with respect to $k$, setting $k=1$, and simplifying, we get

$$
\begin{aligned}
& \int_{0}^{1} \frac{\log (x) \log (\log (x)) \tanh ^{-1}(x)}{x} \mathrm{~d} x \\
& =\frac{1}{8}\left(-7 \zeta^{\prime}(3)+\zeta(3)(-7+7 \gamma-7 i \pi-\log (2))\right),
\end{aligned}
$$

from Example 1 in Section 12.1 in [7].

Example 6. Using equation (16), taking the first partial derivative with respect to $k$, setting $k=2$, and simplifying, we get 


$$
\begin{aligned}
& \int_{0}^{1} \frac{\log ^{2}(x) \log (\log (x)) \tanh ^{-1}(x)}{x} \mathrm{~d} x \\
& =\frac{15 \zeta^{\prime}(4)}{8}+\frac{\pi^{4}(45-30 \gamma+30 i \pi+\log (4))}{1440},
\end{aligned}
$$

from Example 1 in Section 12.1 in [4].

\subsection{Logarithmic Integrals}

Example 7. Using equation (16), taking the first partial derivative with respect to $k$, setting $k=0, a=-1, m=2, n=1$, and simplifying, we get

$$
\begin{aligned}
& \int_{0}^{1} \frac{\log \left((1-x)\left(x^{2}+1\right)\right) \log (\log (x))}{x} d x \\
& =\frac{1}{8}\left(-6 \zeta^{\prime}(2)+(\gamma-i \pi) \pi^{2}\right),
\end{aligned}
$$

from Example 1 in Section 12.1 in [4].

Example 8. Using equation (16), taking the first partial derivative with respect to $k$, setting $k=0, m=n=1$, and simplifying, we get

$$
\int_{0}^{1} \frac{\log \left(1-x^{2}\right) \log (\log (x))}{x} \mathrm{~d} x=\frac{1}{12} \pi^{2}\left(\log \left(\frac{A^{12}}{\pi}\right)-i \pi\right),
$$

from equation (A.11) in [4].

Example 9. Using equation (16), setting $k=1 / 2, m=n=1$, and simplifying, we get

$$
\int_{0}^{1} \frac{\sqrt{\log (x)} \log \left(1-x^{2}\right)}{x} \mathrm{~d} x=-\frac{1}{4} i \sqrt{\frac{\pi}{2}} \zeta\left(\frac{5}{2}\right) .
$$

Example 10. Using equation (16), setting $k=-1 / 2$, $m=n=1$, and simplifying, we get

$$
\int_{0}^{1} \frac{\log \left(1-x^{2}\right)}{x \sqrt{\log (x)}} \mathrm{d} x=i \sqrt{\frac{\pi}{2}} \zeta\left(\frac{3}{2}\right)
$$

Example 11. Using equation (16), taking the first partial derivative with respect to $k$, setting $k=m=n=1$, and simplifying, we get

$$
\begin{aligned}
& \int_{0}^{1} \frac{\log (x) \log \left(1-x^{2}\right) \log (\log (x))}{x} d x \\
& =\frac{1}{4}\left(\zeta^{\prime}(3)+\zeta(3)(1-\gamma+i \pi-\log (2))\right),
\end{aligned}
$$

from Example 1 in Section 12.1 in [5].

Example 12. Using equation (16), taking the first partial derivative with respect to $k$, setting $k=-1 / 2, m=n=1$, and simplifying, we get

$$
\begin{aligned}
& \int_{0}^{1} \frac{\log \left(1-x^{2}\right) \log (\log (x))}{x \sqrt{\log (x)}} \mathrm{d} x \\
& =\sqrt{\frac{\pi}{2}}\left(i \zeta^{\prime}\left(\frac{3}{2}\right)-i \zeta\left(\frac{3}{2}\right)(\gamma-i \pi+\log (8))\right)
\end{aligned}
$$

from Example 1 in Section 12.1 in [5].

\section{Derivation of Definite Integrals of the Logarithmic Function}

Using equation (11), we take the first partial derivative with respect to $m$ and then replace $m$ by $m+1$. Next, we form a second equation by replacing $m$ by $p$ in the new equation. Then, we take the difference of these two new equations simplifying to get

$$
\begin{aligned}
& \int_{0}^{1} \frac{\log ^{k+1}(x)\left(x^{m}-x^{p}\right)}{\left(x^{m+1}+1\right)\left(x^{p+1}+1\right)} \mathrm{d} x=-2^{-k-1}\left(2^{k+1}-1\right) e^{i \pi k / 2} \zeta(k+2) \Gamma \\
& \cdot(k+2)\left(\frac{(i / m+1)^{k}}{(m+1)^{2}}-\frac{(i / p+1)^{k}}{(p+1)^{2}}\right) .
\end{aligned}
$$

Repeating the steps above using equation (12) and simplifying, we get

$$
\begin{aligned}
& \int_{0}^{1} \frac{\log ^{k+1}(x)\left(x^{n}-x^{p}\right)}{\left(x^{n+1}-1\right)\left(x^{p+1}-1\right)} \mathrm{d} x \\
& =e^{i \pi k / 2} \zeta(k+2) \Gamma(k+2)\left(\frac{(i / p+1)^{k}}{(p+1)^{2}}-\frac{(i / n+1)^{k}}{(n+1)^{2}}\right) .
\end{aligned}
$$

\subsection{Some Special Cases}

Example 13. Using equation (31) and applying L'Hopital's rule to the right-hand side as $k \longrightarrow-2$ and simplifying, we get

$$
\int_{0}^{1} \frac{x^{m}-x^{p}}{\left(x^{m+1}+1\right)\left(x^{p+1}+1\right) \log (x)} \mathrm{d} x=\frac{1}{2} \log \left(\frac{m+1}{p+1}\right) .
$$

Example 14. Using equation (32) and setting $k=1$ and simplifying, we get

$$
\int_{0}^{1} \frac{\log ^{2}(x)\left(x^{n}-x^{p}\right)}{\left(x^{n+1}-1\right)\left(x^{p+1}-1\right)} d x=2 \zeta(3)\left(\frac{1}{(n+1)^{3}}-\frac{1}{(p+1)^{3}}\right) .
$$

\section{Table of Integrals}

Table of definite integrals provided in Table 1 
TABLe 1: Table of definite integrals.

\begin{tabular}{|c|c|}
\hline$f(x)$ & $\int_{0}^{1} f(x) \mathrm{d} x$ \\
\hline $\begin{array}{l}\left(\tanh ^{-1}(x) \log ^{k}(x) / x\right) \\
\left(\log ^{k}(x) \log \left(\left(x^{m}+1\right)\left(1-x^{n}\right)\right) / x\right) \\
\left(\left(\pi^{3}-12 \pi \log ^{2}(x)\right) \tanh ^{-1}(x) / x\left(4 \log ^{2}(x)+\pi^{2}\right)^{3}\right) \\
\left(\tanh ^{-1}\left(x^{m}\right) / x\left(a^{2}+\log ^{2}(x)\right)\right) \\
\left(\log (\log (x)) \tanh ^{-1}(x) / x\right) \\
\left(\sqrt{\log (x)} \tanh ^{-1}(x) / x\right) \\
\left(\tanh h^{-1}(x) / x \sqrt{\log (x)}\right) \\
\left(\log (x) \log (\log (x)) \tanh ^{-1}(x) / x\right) \\
\left(\log (x) \log (\log (x)) \tanh ^{-1}(x) / x\right) \\
\left(\log \left((1-x)\left(x^{2}+1\right)\right) \log (\log (x)) / x\right) \\
\left(\log \left(1-x^{2}\right) \log (\log (x)) / x\right) \\
\left(\sqrt{\log (x)} \log \left(1-x^{2}\right) / x\right) \\
\left(\log \left(1-x^{2}\right) / x \sqrt{\log (x)}\right) \\
\left(\log (x) \log \left(1-x^{2}\right) \log (\log (x)) / x\right) \\
\left(\log \left(1-x^{2}\right) \log (\log (x)) / x \sqrt{\log (x)}\right) \\
\left(\log k+1(x)\left(x^{m}-x^{p}\right) /\left(x^{m+1}+1\right)\left(x^{p+1}+1\right)\right) \\
\left(x^{m}-x^{p} /\left(x^{m+1}+1\right)\left(x^{p+1}+1\right) \log (x)\right) \\
\left(\log k x^{k+1}(x)\left(x^{n}-x^{p}\right) /\left(x^{n+1}-1\right)\left(x^{p+1}-1\right)\right)\end{array}$ & $\begin{array}{c}2^{-k-2}\left(2^{k+2}-1\right) e^{i \pi k} \zeta(k+2) \Gamma(k+1) \\
(1 / 2) e^{i \pi k} \zeta(k+2) \Gamma(k+1)\left(\left(2-2^{-k}\right) m^{-k-1}-2 n^{-k-1}\right) \\
(2 C-1 / 16 \pi) \\
(\pi / 4 a) \log \left(a m \Gamma(a m / 2 \pi)^{2} / 2 \pi \Gamma(a m+\pi / 2 \pi)^{2}\right) \\
(1 / 24) \pi^{2}\left(\log \left(16 \pi^{3} / A^{36}\right)+3 i \pi\right) \\
-(1 / 16) i(\sqrt{2}-8) \sqrt{\pi} \zeta(5 / 2) \\
(1 / 4) i(\sqrt{2}-4) \sqrt{\pi} \zeta(3 / 2) \\
(1 / 8)\left(-7 \zeta^{\prime}(3)+\zeta(3)(-7+7 \gamma-7 i \pi-\log (2))\right) \\
\left(15 \zeta^{\prime}(4) / 8\right)+\left(\pi^{4}(45-30 \gamma+30 i \pi+\log (4)) / 1440\right) \\
(1 / 8)\left(-6 \zeta^{\prime}(2)+(\gamma-i \pi) \pi^{2}\right) \\
(1 / 12) \pi^{2}\left(\log \left(A^{12} / \pi\right)-i \pi\right) \\
-(1 / 4) i \sqrt{\pi / 2} \zeta(5 / 2) \\
i \sqrt{\pi / 2} \zeta(3 / 2) \\
-2^{-k-1}\left(2^{k+1}-1\right) e^{i \pi k / 2} \zeta(k+2) \Gamma(k+2)\left(\left((i / m+1)^{k} /(m+1)^{2}\right)-\left((i / p+1)^{k} /(p+1)^{2}\right)\right) \\
(1 / 2) \log (m+1 / p+1) \\
\sqrt{\pi / 2}\left(i \zeta^{\prime}(3 / 2)-i \zeta(3 / 2)(\gamma-i \pi+\log (8))\right) \\
e^{i \pi k / 2} \zeta(k+2) \Gamma(k+2)\left(\left((i / p+1)^{k} /(p+1)^{2}\right)-\left((i / n+1)^{k} /(n+1)^{2}\right)\right)\end{array}$ \\
\hline
\end{tabular}

\section{Discussion}

In this work, the authors looked at deriving definite integrals of combinations of logarithmic functions of complicated arguments and powers and expressed them in terms of the Hurwitz zeta function. One of the interesting properties of these integrals is that, by adding them, we were able to get the integral of the product of the hyperbolic arctangent function and the logarithmic function. The authors formally derived a few integrals in terms of fundamental constants and special functions. One of our goals was to supply a table of definite integrals (Table 1) for easy reading by researchers and to have these results added to existing textbooks.

The results presented were numerically verified for both real and imaginary values of the parameters in the integrals using Mathematica by Wolfram. We considered various ranges of these parameters for real, integer, negative, and positive values. We compared the evaluation of the definite integral to the evaluated special function and ensured agreement.

\section{Conclusion}

In this paper, the authors used our method to evaluate definite integrals using the Hurwitz zeta function. The contour we used was specific to solving integral representations in terms of the Hurwitz zeta function. The author expects that other contours and integrals can be derived using this method.

\section{Data Availability}

No data were used to support this study.

\section{Disclosure}

This paper is also available in preprint at http://export.arxiv. org/abs/2103.03110.

\section{Conflicts of Interest}

The authors declare no conflicts of interest.

\section{References}

[1] R. Reynolds and A. Stauffer, "A method for evaluating definite integrals in terms of special functions with examples," International Mathematical Forum, vol. 15, no. 5, pp. 235-244, 2020.

[2] A. P. Prudnikov, Yu. A. Brychkov, and O. I. Marichev, Integrals and Series, More Special Functions, USSR Academy of Sciences, Moscow, ID, USA, 1990.

[3] M. Abramowitz and I. A. Stegun, Eds., Handbook of Mathematical Functions with Formulas, Graphs, and Mathematical Tables, Dover, New York, NY, USA, 9th edition, 1982.

[4] I. S. Gradshteyn and I. M. Ryzhik, Tables of Integrals, Series and Products, Academic Press, Cambridge, MA, USA, 6th edition, 2000.

[5] M. Jan, K. B. Oldham, and J. Spanier, An Atlas of Functions: With Equator, the Atlas Function Calculator, Springer, Belin, Germany, 2nd edition, 2009.

[6] A. Voros, "Spectral functions, special functions and the selberg zeta function," Communications in Mathematical Physics, vol. 110, no. 3, pp. 439-465, 1987.

[7] E. T. Whittaker and G. N. Watson, A Course in Modern Analysis, Cambridge University Press, Cambridge, UK, 4th ed. edition, 1902. 